\title{
3 Therapiezielfindung bei potenziellen Organspendern
}

\author{
Stefan Meier und Uwe Janssens
}

\subsection{Therapieziele in der Intensivmedizin}

Intensivmedizinische Behandlungen bedürfen wie alle anderen medizinischen Maßnahmen einer Rechtfertigung, die sich aus einer Indikation für die betreffende Behandlungssituation und einer informierten Einwilligung durch den Patienten oder seiner rechtlichen Vertreter ergibt. Gerade in Notfällen besteht dabei häufig die Besonderheit, dass der Patient nicht einwilligungsfähig ist und die Behandlung, zumindest zu Beginn, häufig ohne vorliegende Einwilligung zum Erhalt des Patientenwohles begonnen wird (Bundesgesetzblatt 2013). Diese Besonderheit entbindet die Behandler aber nicht davon, sobald es möglich ist, eine Einwilligung in die - dann meist schon laufende-Therapie einzuholen.

Da der Patient in dieser Phase zumeist weiterhin nicht in der Lage ist, wirksam in die Behandlung einzuwilligen, muss die Diskussion über die vorgeschlagene Therapie häufig mit den rechtlichen Vertretern des Patienten, so diese vom Patienten überhaupt benannt wurden, oder seinen Angehörigen geführt werden. Gegebenenfalls ist es in der Folge, zum Beispiel bei nicht benannten rechtlichen Vertretern, dann auch notwendig, eine gerichtliche Betreuung anzuregen (Biermann 2019; Bundesgesetzblatt 2009).

In jedem Fall ist es geboten, die intensivmedizinische Therapie an den Behandlungspräferenzen und Behandlungszielen des Patienten auszurichten. Es ist daher notwendig, im Austausch mit dem Patienten - und falls mit ihm nicht möglich, seinen rechtlichen Vertretern - eine Klärung und Festlegung 
der in der betreffenden Behandlungssituation gewünschten Therapieziele zu erlangen. Solche Therapiezielgespräche sollten in ruhiger, ungestörter Atmosphäre ergebnisoffen mit allen an der Behandlung beteiligten Personen geführt werden.

Inhaltlich ist es für die Durchführung solcher Gespräche wichtig zu betonen, dass es auch in der Intensivmedizin mehr als ein einzelnes Therapieziel geben kann. Auch wenn es auf den ersten Blick selbstverständlich erscheint, dass die Stabilisierung des Patienten, die Wiederherstellung der kritisch gestörten Organfunktionen und damit die Genesung des Patienten die primären Ziele intensivmedizinischer Therapie sind, können es die Situation oder auch die dargelegten Präferenzen des Patienten bedingen, dass andere Ziele in den Vordergrund treten. So können auch eine Behandlung, die eine Stabilisierung und Verbesserung der Lebensqualität ohne Anspruch einer Genesung, die Symptomkontrolle und die Sterbebegleitung Therapieziele auf einer Intensivstation sein (Neitzke et al. 2019a).

\section{Auch in der Intensivmedizin können Therapieziele unterschiedlich sein. Therapieziele müssen individuell, patientenzentriert und situationsbezogen definiert werden.}

\subsection{Therapiezielfindung bei potenziellen Organspendern}

Wenn bei Patienten mit wahrscheinlichem Todeseintritt aufgrund der $\mathrm{Ce}$ gebenheiten eine Organspende als denkbare Therapieoption in Frage kommt, muss bei den stattfindenden Therapiezielgesprächen deutlich gemacht werden, dass auch eine Organspende ein mögliches Therapieziel sein kann.

Es ist in diesem Fall von großer Bedeutung, die Option einer Organspende nicht verfrüht zu verwerfen. Sofern nicht eindeutige Kontraindikationen gegen eine Organspende sprechen, ist die Beurteilung, ob ein Patient zum Beispiel aufgrund seines Alters oder seiner Vorerkrankungen als Spender in Frage kommt erst zu klären, nachdem Klarheit über die grundsätzliche Spendebereitschaft des Patienten besteht. Nicht nur Ärzte, auch Angehörige, können sich mitunter nicht vorstellen, dass eine Organspende bei vorerkrankten Menschen möglich ist (Otth et al. 2011) und sprechen deshalb einen möglicherweise generell bestehenden Organspendewunsch des Sterbenden bei Therapiezielgesprächen nicht an.

Ebenso sollte das Vorliegen einer Patientenverfügung nicht per se dazu führen, eine Organspende nicht in Erwägung zu ziehen. Es ist in jedem Fall zu prüfen, in welchem Verhältnis die vorliegende Patientenverfügung zu einem 
möglichen Organspendewunsch steht (Zentrale Ethikkommission bei der Bundesärztekammer 2013). Es ist nicht selten, dass der Wunsch zur Therapiebegrenzung gleichzeitig mit einem Organspendewunsch besteht, auch wenn dies nicht ausdrücklich in der Patientenverfügung formuliert ist. Hier muss gemeinsam mit den gesetzlichen Vertretern - sofern diese vom Patienten benannt wurden - ansonsten mit den Angehörigen des Patienten sorgfältig geklärt werden, ob in der konkreten Situation der Wunsch zur Therapiebegrenzung überwiegt oder ob nicht die intensivmedizinischen Maßnahmen eine gewisse Zeit weitergeführt werden sollen, um eine Organspende zu ermöglichen (Duttge u. Neitzke 2015).

\section{Auch bei Vorliegen einer Patientenverfügung sowie bei älteren oder vorerkrankten Patienten darf die Option einer Organspende nicht frühzeitig verworfen werden, sondern sollte im Gegenteil schon zu diesem Zeitpunkt aktiv ins Gespräch gebracht werden.}

In jedem Fall muss den an den Gesprächen Beteiligten immer bewusst sein, dass Entscheidungen zur Organspende bei den Angehörigen lang, zeitweise über Jahre, nachwirken. Offene Fragen, die im Spendeprozess nicht hinreichend thematisiert und beantwortet werden, nehmen dabei über die Zeit zu und können eine wachsende Belastung für die Angehörigen darstellen. Ein sehr interessanter Aspekt ist, dass Angehörige häufiger mit der Entscheidung hadern, einer Organspende nicht zugestimmt zu haben als einer Spende zugestimmt zu haben (Sarti et al. 2018). Zusammen mit der Beobachtung, dass Angehörige, die einer Organspende nicht zugestimmt haben, die Qualität der Kommunikation schlechter beurteilt haben als Angehörige, die einer Spende zugestimmt haben (Kentish-Barnes et al. 2019), wird die große Bedeutung einer offenen, keine Fragen aussparenden Kommunikation mit den Angehörigen zur Ermöglichung einer gelingenden Verarbeitung der Spendeentscheidung deutlich.

Den Gesprächsführenden sollte bewusst sein, dass Entscheidungen zur Organspende bei Patientenangehörigen lange nachwirken können. Eine hohe Qualität der Kommunikation stärkt die Bereitschaft zur Organspende.

\subsection{Re-Evaluation des Therapieziels Organspende}

Therapieziele müssen sowohl mit Blick auf ihre realistische Erreichbarkeit als auch mit Blick auf den Patientenwillen, der sich im Laufe einer Behand- 
lung durchaus ändern kann, reevaluiert werden. Das bekannte Sprichwort: „wer A sagt, muss auch B sagen“, kann bei der Festlegung von Therapiezielen keine Gültigkeit haben, so gilt zum Beispiel, dass das Recht, in eine Behandlung nicht einzuwilligen, nicht nur vor Beginn einer Behandlung besteht, sondern auch währenddessen. Dies ist gerade auch mit Blick auf eine potentielle Organspende zu beachten, da sich auch hier ein Sinneswandel (der allerdings dann auch auf mögliche Gründe kritisch hinterfragt werden sollte) auftreten kann und respektiert werden muss.

\section{Bei veränderter medizinischer Situation oder geändertem Patien- tenwillen müssen Therapieziele, so auch das Therapieziel Organ- spende, reevaluiert und gegebenenfalls angepasst werden.}

Auch aus medizinischen Cründen ist es notwendig, die Erreichbarkeit des Therapieziels Organspende regelmäßig zu reevaluieren. Mit der „Entscheidungshilfe bei erweitertem Behandlungsbedarf auf dem Weg zur Organspende" steht gerade in den Fällen, in denen zur Erhaltung der Homöostase der Organe des potenziellen Spenders Maßnahmen notwendig werden, die über die Weiterführung der bisherigen intensivmedizinischen Therapie hinausgehen, eine Orientierungshilfe zur Verfügung (Neitzke et al. 2019b).

In diesem Positionspapier der DIVI-Sektionen werden fünf Dimensionen identifiziert, die eine Bedeutung für die ethische Zulässigkeit einer Ausweitung des Behandlungsbedarfs haben. Im Einzelnen handelt es sich dabei um die Dimensionen:

- irreversibler Hirnfunktionsausfall

- Organspendewunsch

- Wille zur Therapiebegrenzung

- Eingriffsintensität der erweiterten Behandlungsmaßnahmen

- Wahrscheinlichkeit der erfolgreichen organprotektiven Therapie

Die Ausprägungsgrade dieser Dimensionen können graphisch in einem Netzdiagramm dargestellt werden (s. Abb. 3). Befinden sich die Ausprägungsgrade in den äußeren Segmenten des Diagramms, erscheint die Fortführung oder auch Erweiterung der Intensivmedizin ethisch gerechtfertigt, wenn nicht sogar geboten. Liegen die Ausprägungsgrade graphisch in den inneren Segmenten, wird die Fortführung oder gar Ausweitung der Therapie ethisch fragwürdig.

Zusammengefasst kann die Abklärung der Therapieoption Organspende ein komplexer Vorgang sein, in den viele Personen einbezogen sind bzw. einbezogen werden müssen. Notwendig ist eine gute Zusammenarbeit und 


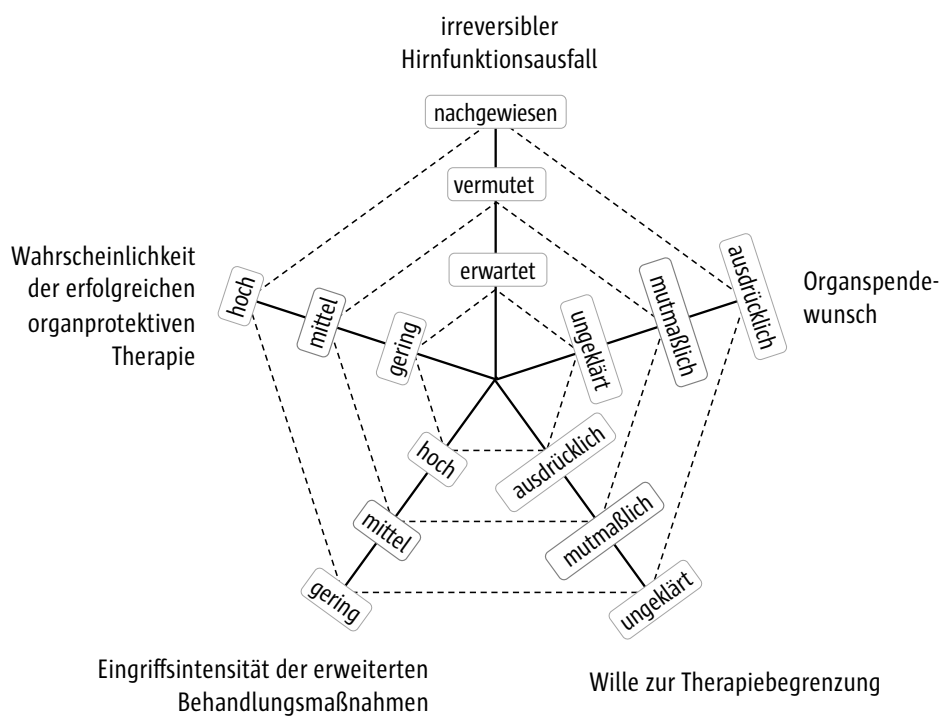

Abb. 3 Netzdiagramm zur Entscheidungsfindung für den erweiterten intensivmedizinischen Behandlungsbedarf auf dem Weg zur Organspende (nach Neitzke et al. 2019b)

Kommunikation zwischen den Mitgliedern des ärztlichen und nicht-ärztlichen Behandlungsteams, den gesetzlichen Vertretern bzw. Angehörigen des Patienten und die Einbeziehung der zuständigen Transplantationsbeauftragten und der Koordinierungsstelle. Es ist sinnvoll und hilfreich, angepasst auf die spezifischen Verhältnisse des jeweiligen Entnahmekrankenhauses, Handlungsempfehlungen zum Beispiel in Form einer Standard Operating Procedure (SOP) zu formulieren. Auf diese Weise werden die Bedingungen optimiert, um eine tragfähige und am Patientenwillen orientierte Entscheidung zur Organspende bei jedem einzelnen potenziellen Organspender zu erreichen.

\section{Literatur}

Biermann E (2019) Patientenverfügung, Vorsorgevollmacht und Betreuung - Aktuelle Urteile des BGH. Anästh Intensivmed. 60: p. 273-283

Bundesärztekammer, Zentrale Ethikkommission bei der Bundesärztekammer (2013) Arbeitspapier zum Verhältnis von Patientenverfügung und Organspendeerklärung. Dtsch Arztebl. 110(12): p. A572A574 
Bundesgesetzblatt (2009) Drittes Gesetz zur Änderung des Betreuungsrechts vom 29. Juli 2009. Bundesgesetzblatt Jahrgang 2009, Teil I Nr. 48, ausgegeben zu Bonn am 31. Juli 2009. URL: http:// www.bgbl.de/xaver/bgbl/start.xav?startbk=Bundesanzeiger_BGBl\&jumpTo=bgbl109s2286.pdf (aufgerufen am 02.06.2021)

Bundesgesetzblatt (2013) Gesetz zur Verbesserung der Rechte von Patientinnen und Patienten vom 20. Februar 2013. Bundesgesetzblatt Jahrgang 2013, Teil I Nr. 9, ausgegeben zu Bonn am 25. Februar 2013. URL: http://www.bgbl.de/xaver/bgbl/start.xav?startbk=Bundesanzeiger_BGBI\&jumpTo=bgbl113s0277.pdf (aufgerufen am 02.06.2021)

Duttge G, Neitzke G (2015) Zum Spannungsfeld zwischen Intensivtherapie und Organtransplantation. DIVI, 6: p. 144-149

Kentish-Barnes N, Siminoff LA, Walker W et al. (2019) A narrative review of family members' experience of organ donation request after brain death in the critical care setting. Intensive Care Med, 45(3): p. $331-342$

Neitzke G, Burchardi H, Duttge G et al. (2019a) Grenzen der Sinnhaftigkeit von Intensivmedizin: Positionspapier der Sektion Ethik der DIVI. Med Klin Intensivmed Notfmed, 114(1): p. 46-52

Neitzke G, Rogge A, Lücking KM et al. (2019b) Entscheidungshilfe bei erweitertem intensivmedizinischem Behandlungsbedarf auf dem Weg zur Organspende: Positionspapier der Sektion Ethik und der Sektion Organspende und -transplantation der Deutschen Interdisziplinaren Vereinigung fur Intensiv- und Notfallmedizin (DIVI) unter Mitarbeit der Sektion Ethik der Deutschen Gesellschaft fur Internistische Intensivmedizin und Notfallmedizin (DGIIN). Med Klin Intensivmed Notfmed, 114(4): p. 319-326

Otth M, Rödder S, Immer FF et al. (2011) Organ donation in Switzerland: a survey on marginal or extended criteria donors (ECD) from 1998 to 2009. Swiss Med Wkly, 141: p. w13230

Sarti AJ, Sutherland S, Healey A et al. (2018) A Multicenter Qualitative Investigation of the Experiences and Perspectives of Substitute Decision Makers Who Underwent Organ Donation Decisions. Prog Transplant, 28(4): p. 343-348 